\title{
Ketorolac Prescribing Practices in an Acute Care Hospital and the Incidence of Acute Renal Failure
}

\author{
Joseph Chan ${ }^{\mathrm{a}, \mathrm{b}}$, Anil Bajnath ${ }^{\mathrm{a}}$, Beth Fromkin ${ }^{\mathrm{a}}$, Debbie Haine ${ }^{\mathrm{a}}$, Rute Paixao ${ }^{\mathrm{a}}$, Dianne Sandy, \\ Umair Rhandhawa $^{\mathrm{a}}$, Fei Wang ${ }^{\mathrm{a}}$, Mauro Braun ${ }^{\mathrm{a}}$
}

\begin{abstract}
Background: Ketorolac has been documented to cause acute kidney injury (AKI) but current data suggest that it is safe for those who have low risk for renal dysfunction. In our facility, there have been cases of AKI in those treated with Ketorolac but the incidence is not known. This study describes the prescribing habits of Ketorolac in our facility and determines the incidence of AKI while on this therapy.
\end{abstract}

Methods: Electronic medical records of patients who received Ketorolac were reviewed during the last 3 months of 2012. AKI was defined as an increase of serum creatinine of $0.3 \mathrm{mg} / \mathrm{dL}$ or greater and a decrease in estimated glomerular filtration rate (eGFR) to less than $60 \mathrm{~mL} /$ minute.

Results: A total of 633 patient charts were reviewed and 341 patients met the inclusion criteria. The mean age was 45.7 years. Sixty-five percent of the patients were females and 35\% were males. The most common diagnosis for prescribing Ketorolac was osteoarthrosis. Thirty milligram IV every 6 hours is the conventional prescribed dose. Of the patients $6.4 \%$ developed AKI during treatment with Ketorolac, $68 \%$ of those with AKI were 65 or older, $68 \%$ had hypertension, $41 \%$ were diabetic, $40 \%$ were concomitantly receiving either an angiotensin converting enzyme-inhibitor (ACE-I) or an angiotensin receptor blocker (ARB), 40\% were also being given diuretics, $72 \%$ received Ketorolac during the time of AKI and $3.8 \%$ of all patients who received Ketorolac developed hyperkalemia while on treatment.

Conclusions: AKI occurs more commonly than previously anticipated in Ketorolac treated patients even at average doses and short durations. Hypertension and diabetes are the two most common co-

Manuscript accepted for publication July 30, 2014

${ }^{a}$ Department of Nephrology and Hypertension, Cleveland Clinic Florida-Weston, 2950 Cleveland Clinic Blvd, Weston, FL 33331, USA

${ }^{b}$ Corresponding Author: Joseph Chan, Department of Nephrology and Hypertension, Cleveland Clinic Florida-Weston, 2950 Cleveland Clinic Blvd, Weston, FL 33331, USA. Email: jchan320@doctor.com

doi: http://dx.doi.org/10.14740/wjnu169w morbidities in patients who developed AKI. Those who are greater than 65 years old may be at higher risk. Concomitant use of drugs that affect renal function, such as ACE-I, ARBs and diuretics, may increase the risk of AKI. Ketorolac prescribing in the acute care hospital should consider individual comorbidities, and use of other drugs that can increase kidney failure risk. Awareness of current renal function through diligent review of daily labs may help prevent administration of Ketorolac in those with impaired renal function. Medication alerts that notifying the ordering physician of the eGFR may help prevent inadvertent prescription in those with AKI or chronic kidney disease.

Keywords: Ketorolac; Acute kidney injury; NSAIDs

\section{Introduction}

Ketorolac (Toradol) is an intravenously administered nonsteroidal anti-inflammatory drug (NSAID) used in clinical practice since the 1990s. Case reports of acute kidney injury (AKI) associated with Ketorolac have been published since then [1-8]. Concerns about other serious side effects such as bleeding diathesis, gastrointestinal complications and even death have been raised $[9,10]$. Current data suggest that its use in patients with normal renal function and when given for less than 5 days is not associated with an increased risk of kidney injury [11]. In addition, IV use of Ketorolac is deemed safe for analgesia even in post-operative patients who have low risk of renal dysfunction $[3,12,13]$. Factors that are known to pose a higher risk for AKI with Ketorolac include conditions such as diabetes, chronic kidney disease (CKD), cirrhosis and heart failure. NSAIDs should be used with caution in those who have concomitant use of other nephrotoxic agents, diuretics, volume depletion and advance age [14].

NSAIDs are potent analgesics with established nephrotoxic potential. They can cause hemo-dynamically mediated renal injury through their inhibitory effects on prostaglandin. NSAIDs can also cause toxicity through interstitial or glomerular disease. Electrolyte disturbances, such as hyperkalemia and hyponatremia are known potential adverse effects 
of NSAIDs [15].

In our facility, Ketorolac is a widely used NSAID type analgesic. It is also incorporated in pain management protocols for post-operative patients. Our Nephrology Consulting Service has noticed increasing incidence of AKI in patients without known renal disease and who received Ketorolac. Furthermore, in those with underlying kidney disease, and in whom Ketorolac was administered, we have seen worsening of their renal function.

Given the widespread use and known side effects of Ketorolac, there is great concern to improve safety of Ketorolac prescription in the hospital setting. Recent data have shown that NSAID prescribing decreased after implementation of estimated glomerular filtration rate (eGFR) reporting [16]. Results of this study can be used to formulate safety protocols and/or laboratory alerts (creatinine and eGFR) through the electronic medical record (EMR) when prescribing Ketorolac. A comparison analysis can be done to evaluate changes in prescribing patterns of Ketorolac after the ordering alert has been implemented. Improvement of NSAID prescribing can also be achieved through educational programs [17]. Results from this study can point to key areas of improvement when prescribing Ketorolac and can be used in educating hospital employees and trainees.

\section{Key questions}

1) How common is Ketorolac used in patients with abnormal renal function in our facility? 2) What is the conventional prescribed dose and duration of Ketorolac? 3) How often is Ketorolac administered to those who have risk for renal injury? (those who have diabetes, CKD, liver disease, heart failure and age $>65$ ). 4) What other potentially nephrotoxic agents are used with Ketorolac? (diuretics, other NSAIDs, angiotensin converting enzyme-inhibitor (ACE-I), angiotensin receptor blockers (ARBs) and vancomycin). 5) What is the incidence of AKI with Ketorolac use in our hospital?

\section{Objectives}

The purpose of this study is to describe Ketorolac prescribing habits of medical professionals in a 150-bed acute care hospital over a 3-month period. The secondary objective is to observe the incidence of AKI in patients who have received Ketorolac.

\section{Patients and Methods}

\section{Study population}

\section{Inclusion criteria}

1) Age 18 and above. 2) Both male and female. 3) Patients who received at least one dose of IV Ketorolac during October 1, 2012 - December 31, 2012 admitted in the Cleveland Clinic Florida Facility. 4) Departments included are: medical ICU, surgical ICU, and medical floors.

\section{Exclusion criteria}

1) Patients in whom IV Ketorolac was prescribed but did not receive the drug. 2) Department excluded: emergency room, PACU, operating room. 3) Patients who were not admitted and who do not have a creatinine level obtained prior to Ketorolac administration. 4) Patients who were not admitted and who do not have a creatinine level obtained at least within the last 3 days of Ketorolac exposure.

\section{Study design}

Retrospective cohort study.

\section{Methods and data collection}

EMRs of patients, who have received IV Ketorolac within October 1, 2012 through December 31, 2012 in the in-patient setting of Cleveland Clinic Florida-Weston, were reviewed. Baseline demographics (age, sex and race), and comorbidities that have been associated with an increased risk of NSAID-induced renal injury (CKD, cardiovascular disease (CVD), hypertension, liver disease and diabetes) were obtained. Data regarding the dose of Ketorolac, pre-dose creatinine, eGFR, and reason for admission, were collected. We also determined if there was a complication of AKI during the treatment duration. Treatment duration was defined as the time of initial administration up to the third day of last exposure to Ketorolac. AKI will be defined as an elevation of serum creatinine by $0.3 \mathrm{mg} / \mathrm{dL}$ and an eGFR of $<60 \mathrm{~mL} /$ $\mathrm{min}$. The presence of hyperkalemia will also be monitored as having serum potassium of greater than 5.3 during the treatment duration.

\section{Results}

A total of 633 patient charts were reviewed and 341 patients met the inclusion criteria. The mean age was 45.7 years. Sixty-five percent of patients were females. The most common diagnosis for prescribing Ketorolac was osteoarthrosis. The conventional prescribed dose is $30 \mathrm{mg}$ IV every $6 \mathrm{~h}$ and the average duration is less than 5 days. During treatment with Ketorolac, $6.4 \%$ of patients developed AKI. In patients with AKI, $74 \%$ were 65 or older, $77 \%$ had hypertension, and $45 \%$ were diabetic. Of the AKI patients, $45 \%$ were concomitantly receiving either an ACE-I or an ARB and $45 \%$ were also being given diuretics. In addition, eight of $22(36 \%)$ patients with AKI received two or more drugs that affect renal func- 
Table 1. Demographics and AKI Risk Factors

\begin{tabular}{|c|c|c|}
\hline Factor & Total patients included $(n=341)$ & Patients with AKI $(n=22)$ \\
\hline Mean age & 45.7 years & 64 years \\
\hline Females & $222(65 \%)$ & $10(45 \%)$ \\
\hline Males & $119(35 \%)$ & $13(55 \%)$ \\
\hline Number of patients with AKI & $23(6.7 \%)$ & $\mathrm{n} / \mathrm{a}$ \\
\hline Hyperkalemia & $13(3.8 \%)$ & $2(9 \%)$ \\
\hline Admitted to ICU & $74(22 \%)$ & $3(14 \%)$ \\
\hline Diabetes & $55(16 \%)$ & $10(45 \%)$ \\
\hline Hypertension & $129(38 \%)$ & $17(77 \%)$ \\
\hline CKD & $4(1.1 \%)$ & $1(5 \%)$ \\
\hline $\mathrm{CHF}$ & $5(1.5 \%)$ & $2(9 \%)$ \\
\hline CVD & $24(7 \%)$ & $2(9 \%)$ \\
\hline Liver disease & $14(4.1 \%)$ & $1(5 \%)$ \\
\hline \multicolumn{3}{|l|}{ Concomitant use } \\
\hline Diuretics & $109(32 \%)$ & $10(45 \%)$ \\
\hline Other NSAIDS & $55(16 \%)$ & $1(4 \%)$ \\
\hline RAS inhibitor (ACE-I or ARB) & $85(25 \%)$ & $10(45 \%)$ \\
\hline Vancomycin & $65(19 \%)$ & $7(32 \%)$ \\
\hline IV contrast & $60(18 \%)$ & $3(14 \%)$ \\
\hline Mean initial rise in creatinine & & $0.6 \mathrm{mg} / \mathrm{dL}$ \\
\hline
\end{tabular}

tion and that can potentiate the AKI risk from NSAIDs. During the time of AKI, 72\% received Ketorolac at least once while eGFR was $<60$. Hyperkalemia occurred in $3.8 \%$ of all patients and in $9 \%$ of those who developed AKI (Table 1).

\section{Discussion}

In this study, we report Ketorolac use and prescription in hospitalized patients in our facility. There are limited data on the incidence of AKI with Ketorolac and its prescription in CKD patients. An Irish study of NSAID prescription practices in 388 patients found that NSAID use was common even in patients with renal risks [18]. Our results show evidence that patients still receive Ketorolac despite AKI. The authors identified $40.3 \%$ CKD patients (St 2-3) and 35.1\% with known ischemic heart disease or hypertension received NSAIDs [18]; however, the incidence of AKI was not determined and the type of NSAID use was varied. Two studies evaluated Ketorolac use on post-surgical patients and found it to be safe without an increased renal failure risk in whom
NSAID was not contraindicated $[12,13]$.

In a previous study, incidence of AKI with Ketorolac use was minor. Feldman et al showed the overall incidence of acute renal failure was $1.1 \%$ [10] compared to ours which was $6.4 \%$. In Feldman's study, definition of AKI was defined as an increase in the serum creatinine of $50 \%$ or greater. We also used the AKIN criteria to define AKI as having $0.3 \mathrm{mg} /$ $\mathrm{dL}$ increase in serum creatinine but also included an eGFR of less than $60 \mathrm{~mL} / \mathrm{min}$.

There were many variations of prescribing Ketorolac by physicians from doses as low as $15 \mathrm{mg}$ once daily up to 30 mg every $6 \mathrm{~h}$. Both scheduled and as needed (PRN) dosing were included even at minimal dose in this study as this can still have adverse renal effects in those already at high risk. The average prescription in most is less than 5 days duration and is in accordance with FDA recommendations.

We found that Ketorolac is used commonly in those who have comorbidities that can increase the risk for NSAIDinduced AKI and other adverse effects. The most common in this study were hypertension, diabetes and admittance to the ICU. FDA recommendations advise that Ketorolac should be 
used with caution in patients with hypertension and taking anti-hypertensive. It can precipitate new onset high blood pressure and worsen existing uncontrolled hypertension. Patients who are on ACE-I, ARBs and diuretics should be monitored closely during treatment with Ketorolac. There was only one patient who received Ketorolac with underlying CKD stage 3. There was an observed worsening of renal function during treatment with Ketorolac in this patient. There were no dialysis patients in this cohort.

Elderly patients are at higher risk for NSAID-induced AKI [14]. The aging kidney is more susceptible to AKI due to anatomical, physiologic and clinical factors. Renal mass is decreased and by age $70,30-50 \%$ of cortical glomerular tissue has been lost [19]. There is an expected age-related decline renal blood flow and eGFR [20]. According to the FDA, the half life of Ketorolac is increased by $5-7 \mathrm{~h}$ in the elderly (65 - 78 years old) compared to their young counterparts and dosage should be adjusted. The elderly also have higher incidence of CHF, HTN, diabetes, CVD and CKD, all of which can increase the risk of AKI. The actual incidence of AKI in these patients may be underestimated because of the blunted rise in serum creatinine due to the decreased muscle mass in geriatrics [21]. Serum creatinine, although not the ideal marker to assess renal function in the elderly, should be used with caution. In our cohort, more than $70 \%$ of those that had AKI were 65 years and older. In addition to above innate risks, it is likely that the elderly have higher need for NSAIDs because of the higher incidence of inflammatory arthritis.

Hyperkalemia was uncommon with Ketorolac use in our patients. Even in the group with AKI, the incidence of hyperkalemia was as low as $9 \%$. The mechanism for which hyperkalemia occurs with Ketorolac use is through decreased potassium excretion through the inhibitory effects of Ketorolac on aldosterone [5]. Hyperkalemia from other etiologies could have contributed. The use of RAS inhibitor, volume depletion resulting in decreased sodium delivery to the distal tubule, use of other NSAIDs or from decreased eGFR itself can likewise cause increase in serum potassium.

The use of drugs that may increase renal failure risk with NSAID was common. Diuretics and RAS inhibitor are the two most frequently prescribed medications along with Ketorolac. We observed that $36 \%$ of those who developed AKI were exposed to two or more potentially nephrotoxic agents further increasing risk of AKI. Vancomycin has been considered to cause kidney injury and $32 \%$ of those with AKI were concomitantly receiving vancomycin and Ketorolac. Recently, we have reported a series of patients who have developed ATN and AIN which was attributed to vancomycin. This antibiotic may have potential nephrotoxic potential and should be used with caution with high-dose NSAIDs.

There are many strengths of this study. First is that it included a general cohort of patients including not only post-operative patients, but also those admitted to the ICU and medical floors. Second, we have included serum creatinine and eGFR to define AKI which makes a more accurate diagnosis especially in the elderly. Also, we were able to highlight the incidence of AKI and more importantly, that the prescription of Ketorolac in those who already have ongoing renal injury is still being given NSAIDs. However, there are several limitations that we recognize. Our study was conducted in a single center and was a retrospective, observational study. The results may not be reflective of other institutions. The audit did not identify patients with early CKD (stage 1-2) because the EMR does not report the actual numerical value of eGFR above $60 \mathrm{~mL} / \mathrm{min}$. In addition, the eGFR calculation did not differentiate between races. Correction of eGFR for ethnicity should be considered in future studies. Comparison with other estimates of renal function such as creatinine clearance was not available. Our findings cannot exclude other causes of AKI such as volume depletion, ATN or other drug-induced AKI. Ketorolac, however, can increase the AKI in susceptible individuals through its inhibitory effect of prostaglandin, risk via mechanisms that affect renal perfusion and decreasing GFR.

We propose that prior to Ketorolac prescription, the renal risk should be identified. The patient's age, serum creatinine and eGFR must be considered and the dose should be adjusted accordingly. PRN, rather than scheduled dosing may be appropriate in most. Review of medications is important in assessing risk. If medications that affect volume, renal dynamics and nephrotoxic agents are being given, Ketorolac should be used with caution and patient's renal function should be monitored closely. An alert notification reporting the current eGFR and serum creatinine may improve prescription of Ketorolac in those with already decreased renal function.

In conclusion, hospitalized patients have characteristics that can increase the risk of AKI with Ketorolac. In our facility, patients who are hypertensive and diabetic are commonly given Ketorolac. Although there was a transient increase in serum creatinine that was observed, there is a higher incidence of AKI in those who receive Ketorolac than previously reported. Concomitant use of diuretics and RAS inhibitors were commonly observed in those who had developed AKI and those who are hypertensive, diabetic or elderly that may be at higher risk.

\section{References}

1. Schoch PH, Ranno A, North DS. Acute renal failure in an elderly woman following intramuscular ketorolac administration. Ann Pharmacother. 1992;26(10):12331236.

2. Corelli RL, Gericke KR. Renal insufficiency associated with intramuscular administration of ketorolac tromethamine. Ann Pharmacother. 1993;27(9):1055-1057. 
3. Buller GK, Perazella MA. Acute renal failure and ketorolac. Ann Intern Med. 1997;127(6):493-494.

4. Boras-Uber LA, Brackett NC, Jr. Ketorolac-induced acute renal failure. Am J Med. 1992;92(4):450-452.

5. Haragsim L, Dalal R, Bagga H, Bastani B. Ketorolacinduced acute renal failure and hyperkalemia: report of three cases. Am J Kidney Dis. 1994;24(4):578-580.

6. Quan DJ, Kayser SR. Ketorolac induced acute renal failure following a single dose. J Toxicol Clin Toxicol. 1994;32(3):305-309.

7. Fong J, Gora ML. Reversible renal insufficiency following ketorolac therapy. Ann Pharmacother. 1993;27(4):510-512.

8. Gales BJ, Gales MA. Death associated with inappropriate ketorolac dosing. Ann Pharmacother. 1995;29(12):1299.

9. Fleming BM, Coombs DW. Bleeding diathesis after perioperative ketorolac. Anesth Analg. 1991;73(2):235.

10. Feldman HI, Kinman JL, Berlin JA, Hennessy S, Kimmel SE, Farrar J, Carson JL, et al. Parenteral ketorolac: the risk for acute renal failure. Ann Intern Med. 1997;126(3):193-199.

11. Le Roux PD, Samudrala S. Postoperative pain after lumbar disc surgery: a comparison between parenteral ketorolac and narcotics. Acta Neurochir (Wien). 1999;141(3):261-267.

12. Acharya M, Dunning J. Does the use of non-steroidal anti-inflammatory drugs after cardiac surgery increase the risk of renal failure? Interact Cardiovasc Thorac Surg. 2010;11(4):461-467.
13. Forrest JB, Camu F, Greer IA, Kehlet H, Abdalla M, Bonnet F, Ebrahim S, et al. Ketorolac, diclofenac, and ketoprofen are equally safe for pain relief after major surgery. Br J Anaesth. 2002;88(2):227-233.

14. Camu F, Lauwers MH, Vanlersberghe C. Side effects of NSAIDs and dosing recommendations for ketorolac. Acta Anaesthesiol Belg. 1996;47(3):143-149.

15. Sterns R. NSAIDs: Electrolyte complications. www.uptodate.com. Jul 17, 2012.

16. Naughton BJ, Singh R, Wisniewski AM, Singh G, Anderson DR. Improving quality of NSAID prescribing by internal medicine trainees with an educational intervention. Teach Learn Med. 2010;22(4):287-292.

17. Wei L, MacDonald TM, Jennings C, Sheng X, Flynn RW, Murphy MJ. Estimated GFR reporting is associated with decreased nonsteroidal anti-inflammatory drug prescribing and increased renal function. Kidney Int. 2013;84(1):174-178.

18. Kitchen J, Kane D. Non-steroidal anti-inflammatory drug prescriptions in hospital inpatients: are we assessing the risks? Ir J Med Sci. 2010;179(3):357-360.

19. Frocht A, Fillit H. Renal disease in the geriatric patient. J Am Geriatr Soc. 1984;32(1):28-43.

20. Fliser D, Zeier M, Nowack R, Ritz E. Renal functional reserve in healthy elderly subjects. J Am Soc Nephrol. 1993;3(7):1371-1377.

21. Stallone G, Infante B, Grandaliano G. Acute kidney injury in the elderly population. J Nephrol. 2012;25(Suppl 19):S58-66. 